\title{
Gene Network for Identifying the Entropy Changes of Different Modules in Pediatric Sepsis
}

\author{
Jing Yang Pingli Zhang Lumin Wang \\ Pediatric Department, Qilu Hospital of Shandong University Qingdao, Qingdao, P.R. China
}

\section{Key Words}

Pediatric sepsis • Protein-protein interactions network (PIN) • Module entropy • Potential gene therapy target

\begin{abstract}
Background/Aims: Pediatric sepsis is a disease that threatens life of children. The incidence of pediatric sepsis is higher in developing countries due to various reasons, such as insufficient immunization and nutrition, water and air pollution, etc. Exploring the potential genes via different methods is of significance for the prevention and treatment of pediatric sepsis. This study aimed to identify potential genes associated with pediatric sepsis utilizing analysis of gene network and entropy. Methods: The mRNA expression in the blood samples collected from 20 septic children and 30 healthy controls was quantified by using Affymetrix HG-U133A microarray. Two condition-specific protein-protein interaction networks (PINs), one for the healthy control and the other one for the children with sepsis, were deduced by combining the fundamental human PINs with gene expression profiles in the two phenotypes. Subsequently, distinct modules from the two conditional networks were extracted by adopting a maximal clique-merging approach. Delta entropy $(\Delta S)$ was calculated between sepsis and control modules. Results: Then, key genes displaying changes in gene composition were identified by matching the control and sepsis modules. Two objective modules were obtained, in which ribosomal protein RPL4 and RPL9 as well as TOP2A were probably considered as the key genes differentiating sepsis from healthy controls. Conclusion: According to previous reports and this work, TOP2A is the potential gene therapy target for pediatric sepsis. The relationship between pediatric sepsis and RPL4 and RPL9 needs further investigation.

(C) 2016 The Author(s)

Published by S. Karger AG, Basel
\end{abstract}

\section{Introduction}

Pediatric sepsis is still one of the major life-threatening diseases in children worldwide $[1,2]$. The incidence of pediatric sepsis is higher in developing country where pathogens

Jing Yang

KARGER
Pediatric Department, Qilu Hospital of Shandong University (Qingdao), No.758 Hefei Road, Qingdao, (P.R.China)

E-Mail jingyoung2016@sina.com 


\section{Cellular Physiology Cell Physiol Biochem 2016;40:1153-1162 \\ \begin{tabular}{l|l|l|} 
and BOI: 10.1159/000453169 & $\begin{array}{l}\text { C) 2016 The Author(s). Published by S. Karger AG, Basel } \\
\text { www.karger.com/cpb }\end{array}$
\end{tabular} \\ Yang et al.: Identifying the Entropy Changes of Difference Module in Pediatric Sepsis}

readily invade children because of low birth weight, water and air pollution as well as insufficient immunization and nutrition [3, 4]. Pediatric sepsis is a spectrum of disorders that mainly infected by bacteria, viruses, fungi, parasites or the toxic byproducts of these microorganisms $[5,6]$. Recently, many epidemiologic data reveal that a majority of severe sepsis attacks children without any predisposing condition [7]. Although the diagnosis and management of sepsis in infants and children is mostly influenced by studies done in adults, many important considerations relevant to pediatrics need regard [8]. For the management of pediatric sepsis, intensive attention should be diverted to the age and immune capacity, site, severity and source of infection [6,8]. Exploring potential genes via different methods is essential for the prevention and treatment of pediatric sepsis.

Genes that differentially expressed between the sepsis and control groups have been mainly identified by computational methods [9]. The bioinformatics analysis method has been widely employed to identify potential pathogenic genes [9]. The expression profiles of potential pathogenic genes are probably similar to or negative with those of known pathogenic genes, which are integral part of disease molecular networks or have disease associations in previous literatures [10]. Cancer genes generally participate in core mechanisms, such as DNA damage repair and cell cycle. Therefore, identification of core modules including pathways and complexes dysregulated in cancer is important to explore novel pathogenic genes [10]. Multi-omics data have been integrated to identify the potential gene therapy target [11]. Somatic mutations and gene expression are correlated with to identify novel genes in glioblastoma multiforma. Gene expression, DNA methylation and microRNA expression data of ovarian cancer samples have been combined and analyzed to identify disrupted pathways [11]. Protein-protein interaction networks (PINs) are important class of biological networks, which are obtained from high-throughput interaction screens as well as literature citation [12]. PINs have been constructed by taking individual proteins as nodes and pair-wise interactions between them as edges [12]. In addition, an interaction enrichment analysis has been used to identify pairs of genes whose relationship differed between the normal and diseased samples [13]. Then, interaction profiles have been categorized as cooperative (expression correlated), competitive (expressions anti-correlated), redundant (suppression of both causes dysfunction) and dependent (expression of one is dependent on the other) [13]. Although existing PINs are largely incomplete, they also could represent crude models of protein interaction [12, 14]. Furthermore, integrating PINs with gene expression data is a novel approach to identify candidate gene modules, which are novel diagnostic or prognostic markers or even drive disease progression [13]. Several obvious virtues of the integrative PIN-mRNA expression approach are that (1) it resolves some of the inherent problems involved with analyzing gene expression data separately, such as poor signal to noise ratios and the nodes of explaining gene expression features, (2) integrative PIN-mRNA expression studies have indeed helped tease out relevant patterns of expression variation in the contextual framework of signaling pathways and protein complexes, (3) combination of PINs and expression correlation networks could distinguish direct and indirect correlations, further remove the (long-distance) indirect correlation between genes [14]. PINs have been applied to explore the disease associated genes and pathways in many literature [15]. Disrupted pathways in glioblastoma multiforma have been identified by tracking back paths through the human PINs from differentially expressed gene (target). To discover potential drug targets genes, PINs and gene expression data have been combined to construct a cancer-perturbed PIN in cervical carcinoma. PINs and gene expression data have been combined to construct tissue-specific PINs for 60 tissues and used them to prioritize disease genes. Recently, identification of specific subnetworks that may be causally involved with disease progression has attracted increasingly attention. A comprehensive analysis of cancer networks have been performed by first selecting genes differentially expressed between cancer and normal tissue (the seeds), and then constructing the network as the nearest-neighbor PIN expansion of these seeds. Therefore, statistical properties of networks underlying the cancer phenotype have been explorated to some degree as mentioned above. 


\section{Cellular Physiology Cell Physiol Biochem 2016;40:1153-1162

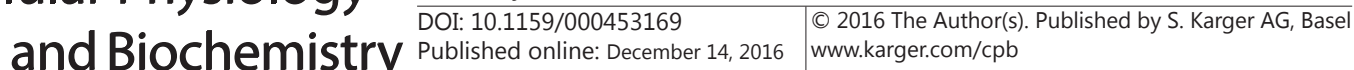 \\ Yang et al.: Identifying the Entropy Changes of Difference Module in Pediatric Sepsis}

Microarray data and genome-wide expression profiling also have been increasingly applied to identify novel pathways and therapeutic gene targets, sepsis-related biomarkers [6, 15-17]. A microarray-based study in children with septic shock demonstrated that development age is a major contributor to sepsis heterogeneity. Chemokine (C-C) motif ligand 4 (CCL4) and interleukin-8 (IL-8) have been identified as outcome biomarker in children with septic shock via analyzing microarray data $[18,19]$. Septic shock subclasses in children have been identified according to analysis of genome-wide expression profiling [20]. Microarray-based studies in children with septic shock have reported high levels of zinc transporter gene SLC39A8 (also known as ZIP8) expression in non-survivors, relative to survivors. Metalloproteinase (MMP)-8 has consistently been the highest expressed gene in children with septic shock relative to normal controls $[19,21]$.

Indeed, approaches based on single-gene differential expression analysis are too simplistic, which have been gradually superseded by network-based methods [10]. Thus, further understanding the statistical properties of the underlying pediatric sepsis networks is critical for better identifying signaling pathways or functional modules involved in disease progression [6]. In this work, network properties that vary significantly between pediatric infected sepsis and normal children were identified by integrating PIN-mRNA expression networks. Particularly, disruptions in the integrated PIN-mRNA expression networks and the randomness of the local correlation patterns were analyzed due to the hypothesis that the frequency of genomic alteration was generally associated with a poor prognosis phenotype [6]. Thus, local and global entropy measure that quantified such randomness was introduced. The results showed that (1) it better characterized the pediatric infected sepsis than other metrics which did not quantify randomness, and (2) the entropy measure helped to unearth potential genes and functional modules associated with the progression of pediatric sepsis.

\section{Materials and Methods}

\section{Study subjects}

In this study, all participants were divided into the control group and pediatric infected sepsis group. Thirty healthy children were assigned in the control group, and a total of 20 children infected with sepsis were allocated into the infected sepsis group. All participants were aged $<14$ years old in this investigation.

\section{Ethics statement}

The procedures of this study were approved by the institutional review board of our hospital. Written informed consents were obtained from all patients and their official guardians.

\section{Extraction of total RNA}

Blood samples were obtained from 20 sepsis infected children and 30 healthy controls. Total RNA was isolated from the whole blood. Initially, the RNA solution was carefully removed using an automatic pipette without disturbing the cell pellet. The cell pellet was washed three times with $7.0 \mathrm{~mL}$ of cold PBS. During the third washing step, the suspension was sonicated with three pulses of $30 \mathrm{~s}$ at $7 \mathrm{~W}$ with 1 min interval on ice (Branson Ultrasonics Co., CT, USA). After the washing-sonication procedure the cell suspension was centrifuged at $5500 \times \mathrm{g}$ at $4^{\circ} \mathrm{C}$ for $10 \mathrm{~min}$ and the pellets were subjected to RNA extraction according to published protocols. Briefly, the pellets were re-suspended in $0.75 \mathrm{ml}$ RNAse-free NAES buffer (50 mM sodium acetate buffer, $10 \mathrm{mM}$ EDTA and 1\% SDS, w/v, pH 5.0) and vortexed for $1 \mathrm{~min}$. The same volume of acid phenol:chloroform (5:1, pH 4.5; Ambion, Inc., Austin, TX, USA) was added to the suspension, then vortexed and transferred to $2.0-\mathrm{ml}$ screw-cap microcentrifuge tubes containing $0.8 \mathrm{~g}$ of glass beads (0.5-mm diameter; Biospec Products, Bartlesville, OK, USA). The cells were lysed in a Mini-Bead Beater homogenizer (Bio-spec Products) at $4^{\circ} \mathrm{C}$ for a total of $120 \mathrm{~s}$ (beat three times for $40 \mathrm{~s}$ with 1 min interval). The homogenized suspension was centrifuged at $10,000 \times \mathrm{g}$ for $5 \mathrm{~min}$ at $4^{\circ} \mathrm{C}$, and the aqueous phase was collected and transferred to a microcentrifuge tube to which was added $0.75 \mathrm{ml}$ of acid phenol:chloroform (5:1, pH 4.5). The tube was vortexed briefly, and centrifuged at $13,000 \times \mathrm{g}$ for $5 \mathrm{~min}$ at $4^{\circ} \mathrm{C}$. The aqueous phase was collected and extracted with a 1:1 solution of chloroform:isoamylalcohol (24:1; Ambion, Inc.) 


\section{Cellular Physiology Cell Physiol Biochem 2016;40:1153-1162

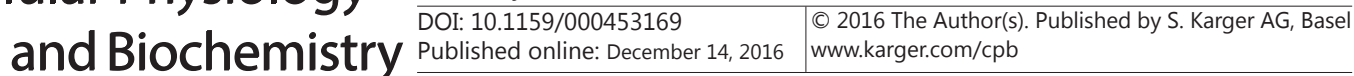 \\ Yang et al.: Identifying the Entropy Changes of Difference Module in Pediatric Sepsis}

once. Total RNA was precipitated using a 1/10 volume of 3 M sodium acetate $(\mathrm{pH}=5)$ and 1 volume of isopropanol at $-20^{\circ} \mathrm{C}$ for at least $30 \mathrm{~min}$.

\section{Establishment of human PPI network via STRING}

The human PINs were downloaded from Search Tool for the Retrieval of Interacting Genes (STRING) database, which was transformed into a standard workable format [22]. To remove redundant interactions, UniProt Knowledgebase (UniProtKB) accession numbers were used as unique protein identifiers. Proteins with names that could not be mapped to UniProtKB accession numbers were discarded [23, 24]. The combined score of each pair-wise interaction was not less than 0.8, and PINs containing 8590 nodes with 53975 documented interactions were obtained.

\section{Gene expression data sets between sepsis infected children and healthy controls}

The mRNA expression was detected by using an Affymetrix HG-U133A microarray. The data were preprocessed and normalized, including background correction via robust multiple-array average, quantile normalization, correcting the perfect match (PM)/mismatch (MM) via MAS 5.0 algorithm, log expression was estimated by an iterative median polishing procedure [25]. Finally, the expression profile containing 13436 genes was obtained. Integrated PIN-mRNA expression networks were constructed from the STRING PIN and the mRNA expression data. The proteins corresponding to gene expression profiles were extracted from the PINs. Thus, the new PINs containing 6132 genes (6132 nodes) with 35184 pair-wise interactions were obtained.

Inferring the PINs between sepsis infected children and healthy controls

Two condition-specific PINs, one for the control group and the other one for sepsis infected group, were constructed based on the human PINs incorporating with gene expression profiles in the two phenotypes [26]. To investigate the differences in network properties between sepsis infected and control groups, correlation expression networks were constructed by separately computing gene pair-wise Pearson correlation across two groups. The Pearson correlation of each pair-wise interaction in two PINs was calculated. The absolution of Pearson correlation of each pair-wise interaction under the normal condition is considered as the combined-score of each edge. Thus, the two conditional PINs with combined-score were inferred by re-weighting the interaction in the generic PINs based on these distributions.

\section{Identifying and pruning modules from the PINS}

The integrated PIN-mRNA networks with the edge weights $p_{i j}$ for two phenotypes (sepsis infected and healthy control) were constructed as described previously. Briefly, edge weights in the PIN are defined by a stochastic matrix $p_{i j}$

$$
p_{i j}=\frac{w_{i j}}{\sum_{k \in \mathcal{N}(i)} w_{i k}}
$$

Where $N_{(i)}$ denotes the neighbors of gene $i$ in the PIN, $w_{i j}=0.5^{*}\left(1+C_{i j}\right)$ denotes the transformed Pearson correlation coefficient $C_{i j}$ of gene expression between genes $i$ and $j$ across the samples belonging to the given phenotype. Whenever $(i, j)$ is not an edge in the PIN, the $p_{i j}$ value was denoted as 0 . The procedure was applied to the two phenotypes, then two integrated PIN-mRNA networks (infected sepsis phenotype $p_{i j(I)}$ and normal phenotype $p_{i j(N)}$ ) were obtained. It is important to point out that the construction of our stochastic matrix means that the topological degrees of each node remain unchanged between the normal and infected sepsis phenotypes.

The module-identification algorithm used in this work is based on clique-merging, similar to the ones previously proposed for identifying complex from PINs. The algorithm works in two steps: finding all maximal clique from PINs and ranking them in descending order of their weighted interaction densities (WID); then, clique-merging approach was applied to construct modules.

The set $C$ of all maximal cliques of size at least $k$ in the PIN was identified via using a fast depthfirst search with pruning-based algorithm [27]. Subsequently, for every clique $c \in C$ we calculate its weight interaction density $(W I D) d_{w}(C)$ as, 


$$
d_{w}(C)=\frac{\sum_{(p, q) \in C} r(p, q)}{\left(\begin{array}{c}
|C| \\
2
\end{array}\right)}
$$

These cliques were ranked in the descending order of their $d_{w}(C)$ values. The ordered list was inspected repeatedly to merge highly overlapping cliques and to build modules. Specifically, for every clique $c_{i}$ in the list, we repeatedly try to find a clique $c_{j}(j>i)$ such that the overlap $\left|c_{i} \cap c_{j}\right| /\left|c_{j}\right| \geq t_{0^{*}} t_{0}$ is a predefined overlapthreshold. If such a $c_{j}$ is found, the weight inter-connectivity $I_{w}$ between the non-overlapping protein of $c_{i}$ and $c_{j}$ were calculated.

$$
I_{w}\left(C_{i}, C_{j}\right)=\frac{\sum_{p \in\left\{C_{i} \backslash C_{j}\right\}, q \in\left\{C_{j} \backslash C_{i}\right\}} r(p, q)}{\left|C_{i} \backslash C_{j}\right| .\left|C_{j} \backslash C_{i}\right|}
$$

If $I_{w}\left(c_{i}, c_{j}\right) \geq t_{m}$, a predefined merge-threshold, then $c_{j}$ is merged into $c_{i}$ forming a module, else $c_{j}$ is discarded.

Comparing modules between infected sepsis and control groups

$R$ and $T$ were defined as the sets of modules identified from the networks $H_{C}$ and $H_{l}$, respectively. For each $R_{i} \in R, T_{j} \in T$, their module correlation density $d_{c c}\left(R_{j}\right)$ and $d_{c c}\left(T_{j}\right)$ was calculated respectively as previous literature [26]. A similarity graph $M=\left(V_{M^{\prime}} E_{M}\right)$ were built, where $V_{M}=\{R \cup T\}$, and $E_{M}=\cup\left\{\left(R_{p} T_{j}\right): J\left(R_{p} T_{j}\right) \geq t_{\rho}\right.$ $\left.\Delta_{c c}\left(R_{i} T_{j}\right) \geq \delta\right\}$, where $J\left(R_{i}, T_{j}\right)=\left|R_{i} \cap T_{j}\right| / \mid R_{i} \cup T_{j} /$ is the Jaccard similarity and $\Delta_{c c}\left(R_{i} T_{j}\right)=\mid d_{c c}\left(R_{j}\right)-d_{c c}\left(T_{j}\right) /$ is the differential correlation density between $R_{i}$ and $T_{j}, t_{j}$ and $\delta$ are thresholds [26]. Every edge $\left(R_{i}, T_{j}\right)$ is weighted by $J\left(R_{r}, T_{j}\right)$. Then, the disrupted module pairs $\Gamma(R, T)$ were identified by finding the maximum weight matching in $M$ [26]. Finally, they were ranked in descending order of their differential density $\Delta_{c c^{*}}$

To identify altered modules, thresholds of $t_{J}$ was set high, which ensured that the module pairs either had the same gene composition or lost or gained several genes (e.g. if $\left|R_{i}\right|=8,\left|T_{j}\right|=9$, then $t_{J}=2 / 3$ required at least an overlap of 7). Among these, the module pairs with higher differential correlation were ranked higher.

The global entropy of each module

Entropy, which quantified the amount of randomness/disorder in the local flux distribution surrounding any given node $i$, was measured $[14,28]$. The objective modules consisted of the common genes existed between control module and infected sepsis module. $S_{i}$ denotes the local entropy of the node $i$,

$$
S_{i}=-\frac{1}{\log k_{i}} \sum_{j \in \mathcal{N}(i)} p_{i j} \log p_{i j}
$$

Where $k_{i}$ represents the degree of node $i$. Local entropy of each node in the two modules was calculated. Furthermore, the global entropy $\left(S_{I}\right.$ and $\left.S_{C}\right)$ between two modules and $\Delta S\left(\Delta S=S_{I}-S_{C}\right)$ were obtained. The globe entropy $S$ was calculated according to the following equation:

$$
S=\sum_{i=1}^{n} C_{i} S_{i}
$$

Where n represents the number of nodes, $C_{i}\left(C_{i}=k_{i} /(n-1)\right)$ represents the degree centrality of node $i$. Thus, the global entropies of infected sepsis and normal samples $\left(S_{I}\right.$ and $\left.S_{C}\right)$ were calculated, and $\Delta S(\Delta S=$ $S_{I}-S_{C}$ ) were obtained.

\section{Results and Discussion}

\section{Analyzing disruptions of PINs and modules in pediatric sepsis}

The control $H_{C}$ and infected sepsis $H_{I}$ networks displayed an equal quantity of pair-wise interactions, 18710 pair-wise interactions (also named as maximal cliques) with average scores (weight interaction density) of 0.150 and 0.139 , respectively. Among these pair-wise interactions, both $H_{C}$ and $H_{I}$ networks had 5432 maximal cliques when the number of nodes limited in the range $4 \leq \mathrm{n} \leq 20$. After merging highly overlapping cliques, infected sepsis group and control group obtained 25 and 9 modules, respectively. As illustrated in Table 1, 25 


\section{Cellular Physiology Cell Physiol Biochem 2016;40:1153-1162 \begin{tabular}{ll|l} 
DOI: 10.1159/000453169 & $\begin{array}{l}\text { O 2016 The Author(s). Published by S. Karger AG, Basel } \\
\text { www.karger.com/cpb }\end{array}$
\end{tabular} \\ Yang et al.: Identifying the Entropy Changes of Difference Module in Pediatric Sepsis}

Table 1. Illustration of 25 modules obtained in infected sepsis group and genes in each module

\begin{tabular}{ll}
\hline $\begin{array}{l}\text { No. of } \\
\text { module }\end{array}$ & Genes in each module \\
\hline 1 & CENPM,NDC80,CDCA3,NCAPG,NUSAP1,CDC45,CDCA5 \\
2 & FBX05,CCNB1,NCAPG,GMNN,CDC45,MCM4,TYMS \\
3 & TRIP13,CDC45,NCAPG,NDC80,CCNB1,KIF11,TTK,DLGAP5,OIP5,NUSAP1,CDCA3,RAD51AP1,TOP2A \\
4 & KIF22,AURKA,CDCA3,NCAPG,KIF11,CDCA5 \\
5 & MCM5,MCM4,CDC45,MCM3,LIG1,FEN1 \\
6 & CEBPZ,NIP7,WDR12,EBNA1BP2,NOP58 \\
7 & AURKA,CCNB1,NDC80,NCAPG,PCNA,KIF23,MCM4,CDC45 \\
8 & MCM5,MCM4,CDC45,NCAPG,POLE2,MCM7,KIF11 \\
9 & TRIP13,CDC45,ASF1B,MCM4,AURKB \\
10 & RPL18,RPS16,RPL35,RPL8,RPL10,RPS13,RPS5 \\
11 & RPS20,RPS13,RPS16,RPS19,RPS5,RPL35,RPL8,RPL6,RPL18A,RPS27A,RPL19 \\
12 & POLE2,MCM4,CDC45,DLGAP5,NCAPG,NDC80,GINS2,RFC4 \\
13 & FBX05,HELLS,NCAPG,NDC80,KIF11,TTK,KIF23,CDC45,MCM4,CCNA2 \\
14 & TRIP13,CDC45,MCM3,MCM4,CCNB1,NDC80,GINS2,BUB1 \\
15 & EIF3E,EEF1B2,RPL35,RSL24D1,RPL9 \\
16 & TRIP13,CDC45,ASF1B,CDCA3,MCM5,CDCA5 \\
17 & KRR1,BYSL,NAT10,MPHOSPH10,NIFK \\
18 & MND1,CDK4,CCNB1,CDC45,RFC4 \\
19 & TIMELESS,TIPIN,CDC45,MCM4,MCM3,PCNA \\
20 & MCM5,MCM4,CDC45,ASF1B,RNASEH2A,MCM2 \\
21 & MCM5,MCM4,CDC45,MCM3,CCNB1,GMNN,CDC7,RFC4 \\
22 & OIP5,NDC80,CENPO,CENPK,BUB1 \\
23 & RPA3,CDC45,CDK4,CDC7,PCNA \\
24 & MCM3,CCNE1,CDKN1A,CDC45,CCNA2 \\
25 & RBM28,CEBPZ,NIP7,DDX56,NIFK \\
\hline & \\
\hline
\end{tabular}

Table 2. Illustration of 9 modules obtained in control group and genes in each module

\begin{tabular}{|c|c|}
\hline $\begin{array}{l}\text { No. of } \\
\text { module }\end{array}$ & Genes in each module \\
\hline 1 & POLE2,MCM4,CDC45,TTK,NCAPG,KIF11,NDC80,DLGAP5,CCNA2 \\
\hline 2 & TRIP13,CDC45,NCAPG,NDC80,CCNB1,KIF11,TTK,DLGAP5,HJURP,CDCA3,NUSAP1,AURKA \\
\hline 3 & RPS20,RPS13,RPS16,RPS19, RPS5,RPS18 \\
\hline 4 & EIF3E,EEF1B2,RPL35,RSL24D1,RPL4 \\
\hline 5 & RPS5, RPL35,EEF1B2,RPS19, RPS13,RPL6,RPL18A, RPL27, EIF3E, RPL7A \\
\hline 6 & $\mathrm{FBXO5,CCNB1,NCAPG,GMNN,CDC45,MCM4,CCNB2}$ \\
\hline 7 & RPL18,RPS16,RPL35,RPL8,RPL10,RPS13,RPS5 \\
\hline 8 & FBXO5, HELLS, NCAPG, NDC80, GINS2,CDC45, NUSAP1, RRM2 \\
\hline 9 & RPS20,RPS13,RPS16,RPL36AL,RPL18A,RPL19,RPL35,RPL8 \\
\hline
\end{tabular}

Table 3. Jaccard similarity (J score $>0.25)$ and differential correlation density $(\triangle \mathrm{CC})$ between the control and sepsis modules (The nodes are not less than 4)

\begin{tabular}{|c|c|c|c|}
\hline $\begin{array}{l}\text { No. of sepsis } \\
\text { module }\end{array}$ & $\begin{array}{l}\text { No. of control } \\
\text { module }\end{array}$ & I score & $\Delta_{\mathrm{CC}}$ \\
\hline 10 & 7 & 1 & 0.0374 \\
\hline 2 & 6 & 0.75 & 0.0696 \\
\hline 15 & 4 & 0.667 & 0.0244 \\
\hline 3 & 2 & 0.667 & 0.0469 \\
\hline 13 & 1 & 0.583 & 0.0151 \\
\hline 11 & 9 & 0.583 & 0.0412 \\
\hline 12 & 1 & 0.545 & 0.0161 \\
\hline 8 & 1 & 0.454 & 0.0232 \\
\hline 11 & 3 & 0.417 & 0.0218 \\
\hline 11 & 5 & 0.4 & 0.0330 \\
\hline 13 & 8 & 0.385 & 0.0336 \\
\hline 11 & 7 & 0.385 & 0.0340 \\
\hline 3 & 1 & 0.375 & 0.0433 \\
\hline 7 & 6 & 0.364 & 0.0412 \\
\hline 10 & 9 & 0.364 & 0.0445 \\
\hline 1 & 8 & 0.364 & 0.0846 \\
\hline 1 & 2 & 0.358 & 0.0697 \\
\hline 7 & 2 & 0.333 & 0.0280 \\
\hline 12 & 8 & 0.333 & 0.0346 \\
\hline 7 & 1 & 0.308 & 0.0244 \\
\hline 13 & 6 & 0.308 & 0.0318 \\
\hline 10 & 3 & 0.3 & 0.0250 \\
\hline 13 & 2 & 0.295 & 0.0187 \\
\hline 4 & 2 & 0.286 & 0.0422 \\
\hline 8 & 6 & 0.273 & 0.0399 \\
\hline 14 & 2 & 0.25 & 0.0170 \\
\hline 12 & 2 & 0.25 & 0.0197 \\
\hline
\end{tabular}

modules and corresponding genes in the infected sepsis group were revealed and 9 modules and corresponding genes in the control group were illustrated in Table 2.

Next, a comparative analysis of normal $R$ and infected sepsis $T$ modules was performed to understand disruption at the module level. Twenty seven HD modules were obtained, of which the J score $>0.25, \Delta_{c c}>0.01$ and $\mathrm{n} \geq 4$. The $27 \mathrm{HD}$ modules and their $\mathrm{J}$ scores as well as $\Delta_{c c}$ were listed in Table 3. The local entropy of each node in normal and infected sepsis modules were calculated, and the global entropy of each normal and infected sepsis modules were obtained based on the local entropy of each node. Thus, the delta entropy $(\Delta S)$ and $p$ values were calculated. Two new objective module were obtained with $p<0.05$. One was the new objective module 3 which was reassembled by infected sepsis module 15 and control module 4 , another was the new objective module 13 which was reassembled by infected sepsis module 3 and control module 1 reassemble $(p<0.05)$. The corresponding genes in the new objective module 3 and 13 with corresponding $\Delta S$ and $p$ values were listed in the Table 4. Figure 1 showed that the entropy and $\mathrm{p}$ values of the new objective modules 3 and 13 ( $p$ $<0.05)$. The new objective module 3 consisted of four genes EIF3E, EEF1B2, RSL24D1 and RPL35. As illustrated in Fig. 2, the new objective module 3 consisted of 4 nodes and 6 edges. Infected module 15 comprised 5 genes EIF3E, EEF1B2, RPL35, RSL24D1 and RPL9, and control 


\section{Cellular Physiology Cell Physiol Biochem 2016;40:1153-1162 \begin{tabular}{cc|cc} 
DOI: 10.1159/000453169 & O 2016 The Author(s). Published by S. Karger AG, Basel \\
and Biochemistry & Published online: December 14, 2016 & mww.kargercom/cpb
\end{tabular} \\ Yang et al.: Identifying the Entropy Changes of Difference Module in Pediatric Sepsis}

Table 4. Genes and corresponding $\Delta \mathrm{S}$ and $\mathrm{p}$ values in the new objective module 3 and 13

\begin{tabular}{llll}
\hline New objective module & Delta entropy $(\Delta S)$ & $p$ value & Genes in each module \\
\hline 3 & 0.069 & 0.004 & EIF3E, EEF1B2, RSL24D1, RPL35 \\
13 & 0.134 & 0.027 & TTK, CDC45, NCAPG, NDC80, KIF11, DLGAP5 \\
\hline
\end{tabular}

module 4 also consisted of 5 genes EIF3E, EEF1B2, RPL35, RSL24D1 and RPL4. The gene RPL4 in control module 4 was replaced by RPL9, which might explain why children who were readily infected sepsis. The J score $=0.667$ was higher than 0.25 , suggesting that the infected module 15 and control module 4 were similar. The new objective module 13 consisted of 6 genes CDC45, TTK, NCAPG, KIF11, NDC80 and DLGAP5. Figure 3 showed the new objective module 13 with 6 nodes and 15 edges. Infected module 3 comprised 13 genes TRIP13, CDC45, NCAPG, NDC80, CCNB1, KIF11, TTK, DLGAP5, OIP5, NUSAP1, CDCA3, RAD51AP1 and TOP2A, and control module 1 consisted of 9 genes POLE2, MCM4, CDC45, TTK, NCAPG, KIF11, NDC80, DLGAP5 and CCNA2. The J score $=0.375$ was higher than 0.25 , indicating that the infected module 3 resembled the control module 1 and that the modules pairs either had the same gene composition or have lost or gained only a few genes. The $\Delta S$ of the new objective modules 3 and 13 were 0.069 and 0.134 . The $p$ values of the new objective modules 3 and 13 were 0.004 and 0.027 , both of which were less than 0.05 .

Modules with changes in gene composition

Gene composition was changed in the two remaining modules. The genes of normal module 4 (EIF3E, EEF1B2, RPL35, RSL24D1 and RPL4) changed to (EIF3E, EEF1B2, RPL35, RSL24D1 and RPL9) in the infected sepsis with RPL4 replaced by RPL9, and the module correlation was increased by 0.024 . RPL4 and RPL9 genes encoded ribosomal proteins which were components of the $60 \mathrm{~S}$ subunit [29]. Ribosomes, the organelles that catalyze protein synthesis, consist of a small $40 \mathrm{~S}$ subunit and a large 60S subunit [29]. RPL4 and RPL9 are related to infectious disease. Sepsis is the systemic response to infectious insult [7]. Despite physiologic changes that occur with maturation, the sepsis-induced failure of the mechanisms

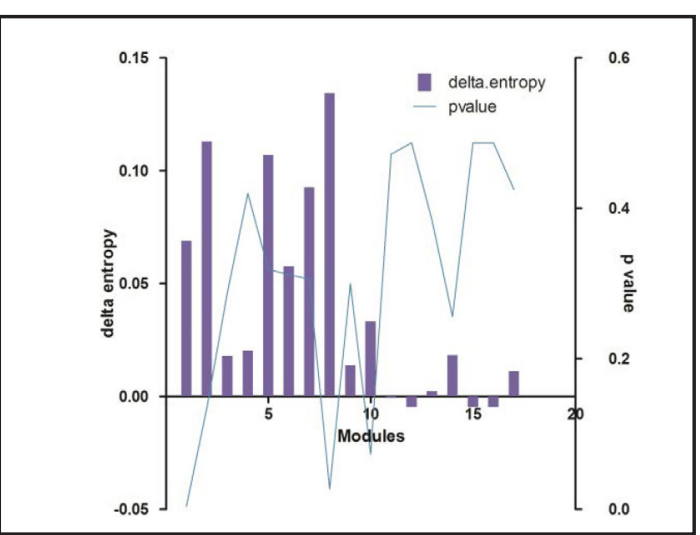

Fig. 1. Illustration of the entropy and $p$ values of the new modules $(p<0.5)$.

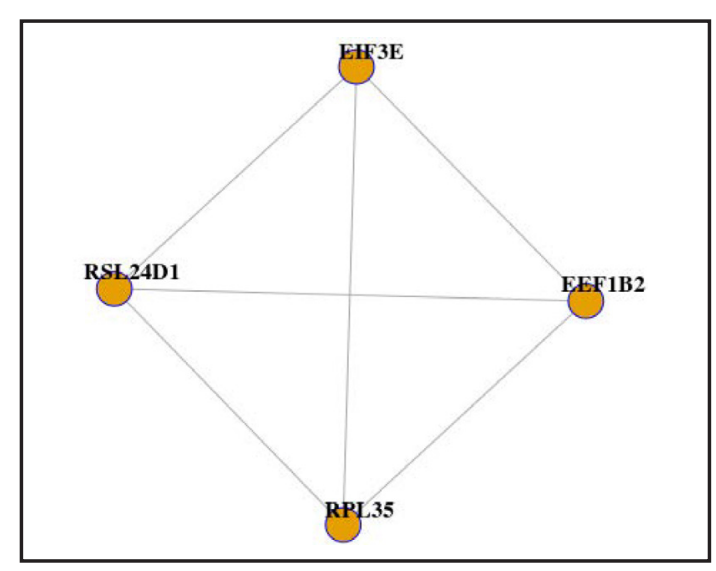

Fig. 2. Analysis of the protein-protein interaction network for infected sepsis children. The network has 4 nodes and 4 edges with $p=0.004$.

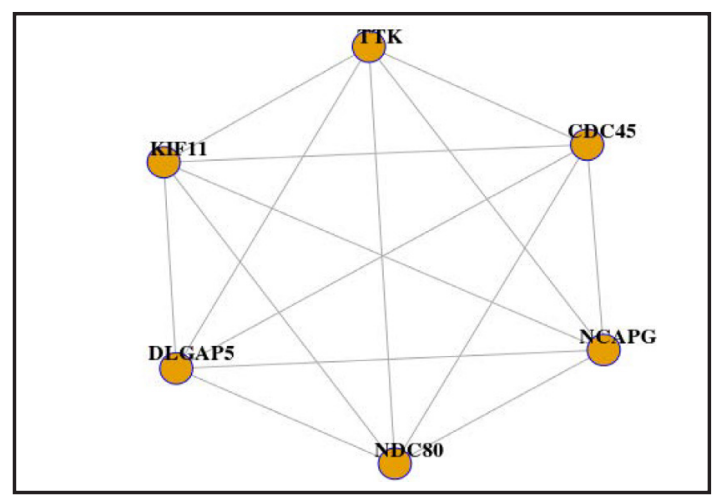

Fig. 3. Analysis of the protein-protein interaction network for infected sepsis children. The network has 6 nodes and 15 edges with $p=0.027$. 


\section{Cellular Physiology Cell Physiol Biochem 2016;40:1153-1162 and BiOChemistry \begin{tabular}{l|l} 
DOI: 10.1159/000453169 & $\begin{array}{l}\text { (c) } 2016 \text { The Author(s). Published by S. Karger AG, Basel } \\
\text { www.karger.com/cpb }\end{array}$
\end{tabular} \\ Yang et al.: Identifying the Entropy Changes of Difference Module in Pediatric Sepsis}

that regulate muscle mass during critical illness have been studied only in adult and mature organisms [30]. To support the systemic inflammatory response, cells with sepsis syndrome mobilize the muscle protein to supply nitrogen [30,31]. Experimental sepsis in mature rats reduces muscle protein synthesis (MPS) by depressing translation of mRNA into protein [30, 32]. The enhanced translational process in the neonatal period significantly declines along with the development of animals due to decreased activation of the translational machinery and reduction of the abundance of the signaling proteins that regulate translation [30]. As mentioned above, RPL4 and RPL9 are probably related to sepsis via regulating the synthesis of ribosomal proteins. However, the direct relationship between RPL4 and RPL9 and sepsis has not been reported, which remains to be further elucidated.

The gene DNA topoisomerase alpha (TOP2A) encodes a DNA topoisomerase, which is an enzyme that controls and alters the topologic states of DNA during transcription [33, 34]. This nuclear enzyme is involved in multiple processes including chromosome condensation, chromatid separation, and the relief of torsional stress that occurs during DNA transcription and replication [33-41]. Protein-protein interaction network of acute respiratory distress syndrome (ARDS) analysis in previous work revealed 20 hub genes including cyclin B1 (CCNB1), cyclin B2 (CCNB2) and TOP2A [42]. Genes including CCNB1, CCNB2 and TOP2A, as well as transcription factors like FOXM1 might be used for novel gene therapy targeting for sepsis-related ARDS [42]. Infected sepsis group contains TOP2A was observed in the infected sepsis group rather than the control group. Therefore, TOP2A is probably the key gene which differentiates the children infected with sepsis from healthy controls. This work further demonstrates that TOP2A is the potential gene therapy target for pediatric sepsis.

\section{Conclusions}

Two condition-specific PINs, one for the healthy controls and the other one for infected sepsis, were inferred by using the human PIN as a backbone and incorporating expression profiles of genes in the two conditions. Subsequently, distinct modules from two conditional networks were extracted by adopting a maximal clique-merging approach. Delta entropy $(\Delta S)$ was calculated between infected sepsis modules and control modules. Then, key genes displaying changes in gene composition were identified by matching control and infected pediatric sepsis modules. Potential roles of these genes in sepsis infected were evaluated according to various references. Therefore, the results showed that TOP2A was the potential gene therapy target for pediatric sepsis. The relationship between pediatric sepsis and RPL4 and RPL9 remains to be further elucidated.

This is a preliminary investigation to initially assess the feasibility of establishing these modules for clinical differential diagnosis of sepsis children from healthy controls. The findings in current study have demonstrated these modules are tentatively applicable for the small sample size investigation (20 sepsis children and 30 healthy controls). To validate the clinical efficacy of these modules for differential diagnosis and treatment of pediatric sepsis, multi-center prospective clinical trials with a larger sample size are urgent to be implemented.

\section{Disclosure Statement}

No.

\section{References}

1 Czaja AS, Zimmerman JJ, Nathens AB: Readmission and late mortality after pediatric severe sepsis. Pediatrics 2009;123:849-857.

2 Odetola FO, Gebremariam A, Freed GL: Patient and hospital correlates of clinical outcomes and resource utilization in severe pediatric sepsis. Pediatrics 2007;119:487-494. 


\section{Cellular Physiology Cell Physiol Biochem 2016;40:1153-1162 \begin{tabular}{l|l|l} 
and Biochemistry $\begin{array}{l}\text { DOI: 10.1159/000453169 } \\
\text { Published online: December 14, } 2016\end{array}$ & $\begin{array}{l}\text { (c) } 2016 \text { The Author(s). Published by S. Karger AG, Basel } \\
\text { www.karger.com/cpb }\end{array}$
\end{tabular} \\ Yang et al.: Identifying the Entropy Changes of Difference Module in Pediatric Sepsis}

3 Wiens MO, Kumbakumba E, Kissoon N, Ansermino JM, Ndamira A, Larson CP: Pediatric sepsis in the developing world: challenges in defining sepsis and issues in post-discharge mortality. Clin Epidemiol 2012;4:319-325.

4 Santiago CD, Menezes AM, de Carvalho WB, Delgado AF: Epidemiologic challenges in sepsis to the developing countries. Pediatr Crit Care Med 2013;14:336.

5 Wong HR: Genome-wide expression profiling in pediatric septic shock. Pediatr Res 2013;73:564-569.

6 Cao $\mathrm{Z}$ and Robinson RA: The role of proteomics in understanding biological mechanisms of sepsis. Proteomics Clin Appl 2014;8:35-52.

7 Martin GS: Sepsis, severe sepsis and septic shock: changes in incidence, pathogens and outcomes. Expert Rev Anti Infect Ther 2012;10:701-706.

8 Randolph, AG and McCulloh RJ: Pediatric sepsis: important considerations for diagnosing and managing severe infections in infants, children, and adolescents. Virulence 2014;5:179-189.

9 Tarca AL, Romero R, Draghici S: Analysis of microarray experiments of gene expression profiling. Am J Obstet Gynecol 2006;195:373-388.

10 Moreau Y, Tranchevent LC: Computational tools for prioritizing candidate genes: boosting disease gene discovery. Nat Rev Genet 2012;13:523-536.

11 Nitsch D, Gonçalves JP, Ojeda F, de Moor B, Moreau Y: Candidate gene prioritization by network analysis of differential expression using machine learning approaches. BMC Bioinformatics 2010;11:460.

12 Prasad TS, Kandasamy K, Pandey A: Human Protein Reference Database and Human Proteinpedia as discovery tools for systems biology. Methods Mol Biol 2009;577:67-79

13 Liu Y, Koyutürk M, Barnholtz-Sloan JS, Chance MR: Gene interaction enrichment and network analysis to identify dysregulated pathways and their interactions in complex diseases. BMC Syst Biol 2012;6:65.

14 Teschendorff AE, Severini S: Increased entropy of signal transduction in the cancer metastasis phenotype. BMC Syst Biol 2010;4:104.

15 Wong HR: Clinical review sepsis and septic shock--the potential of gene arrays. Wong Critical Care 2012;16:204.

16 Wong, H.R., Cvijanovich N, Allen GL, Lin R, Anas N, Meyer K, Freishtat RJ, Monaco M, Odoms K, Sakthivel B, Shanley TP; Genomics of Pediatric SIRS/Septic Shock Investigators: Genomic expression profiling across the pediatric systemic inflammatory response syndrome, sepsis, and septic shock spectrum. Crit Care Med 2009;37:1558-1566.

17 Wong HR, Cvijanovich NZ, Allen GL, Thomas NJ, Freishtat RJ, Anas N, Meyer K, Checchia PA, Lin R, Shanley TP, Bigham MT, Wheeler DS, Doughty LA, Tegtmeyer K, Poynter SE, Kaplan JM, Chima RS, Stalets E, Basu RK, Varisco BM, Barr FE: Validation of a gene expression-based subclassification strategy for pediatric septic shock. Crit Care Med 2011;39:2511-2517.

18 Nowak JE, Wheeler DS, Harmon KK, Wong HR: Admission chemokine (C-C motif) ligand 4 levels predict survival in pediatric septic shock. Pediatr Crit Care Med 2010;11:213-216.

19 Wong HR, Cvijanovich N, Wheeler DS, Bigham MT, Monaco M, Odoms K, Macias WL, Williams MD: Interleukin-8 as a stratification tool for interventional trials involving pediatric septic shock. Am J Respir Crit Care Med 2008;178:276-282.

20 Wong HR, Cvijanovich N, Lin R, Allen GL, Thomas NJ, Willson DF, Freishtat RJ, Anas N, Meyer K, Checchia PA, Monaco M, Odom K, Shanley TP: Identification of pediatric septic shock subclasses based on genome-wide expression profiling. BMC Med 2009;7:34.

21 Wong HR, Shanley TP, Sakthivel B, Cvijanovich N, Lin R, Allen GL, Thomas NJ, Doctor A, Kalyanaraman M, Tofil NM, Penfil S, Monaco M, Tagavilla MA, Odoms K, Dunsmore K, Barnes M, Aronow BJ; Genomics of Pediatric SIRS/Septic Shock Investigators: Genome-level expression profiles in pediatric septic shock indicate a role for altered zinc homeostasis in poor outcome. Physiol Genomics 2007;30:146-155.

22 Szklarczyk D, Franceschini A, Kuhn M, Simonovic M, Roth A, Minguez P, Doerks T, Stark M, Muller J, Bork P, Jensen LJ, von Mering C: The STRING database in 2011: functional interaction networks of proteins, globally integrated and scored. Nucleic Acids Res 2011;39(Database issue):D561-568.

23 Wang L, Yang L, Peng Z, Lu D, Jin Y, McNutt M, Yin Y: cisPath an RBioconductor package for cloud users for visualization and management of functional protein interaction networks. BMC Syst Biol DOI:10.1186/1752-0509-9-S1-S1. 


\section{Cellular Physiology Cell Physiol Biochem 2016;40:1153-1162 \begin{tabular}{ll|l} 
DOI: 10.1159/000453169 & $\begin{array}{l}\text { O 2016 The Author(s). Published by S. Karger AG, Basel } \\
\text { wwww.karger.com/cpb }\end{array}$
\end{tabular} \\ Yang et al.: Identifying the Entropy Changes of Difference Module in Pediatric Sepsis}

24 UniProt Consortium: Update on activities at the Universal Protein Resource (UniProt) in 2013. Nucleic Acids Res 2013;41(Database issue):D43-47.

25 Shippy R, Fulmer-Smentek S, Jensen RV, Jones WD, Wolber PK, Johnson CD, Pine PS, Boysen C, Guo X, Chudin E, Sun YA, Willey JC, Thierry-Mieg J, Thierry-Mieg D, Setterquist RA, Wilson M, Lucas AB, Novoradovskaya N, Papallo A, Turpaz Y, Baker SC, Warrington JA, Shi L, Herman D: Using RNA sample titrations to assess microarray platform performance and normalization techniques. Nat Biotechnol 2006;24:1123-1131.

26 Srihari S, Ragan MA: Systematic tracking of dysregulated modules identifies novel genes in cancer. Bioinformatics 2013;29:1553-1561.

27 Tomita E, Tanaka A, Takahashi H: The worst-case time complexity for generating all maximal cliques and computational experiments. Theor Comput Sci 2006;363:28-42.

28 Jin S, Li Y, Pan R, Zou X: Characterizing and controlling the inflammatory network during influenza A virus infection. Sci Rep 2014;4:3799.

29 Brodersen DE, Nissen P: The social life of ribosomal proteins. FEBS J 2005;272:2098-2108.

30 Orellana RA1, Wilson FA, Gazzaneo MC, Suryawan A, Davis TA, Nguyen HV: Sepsis and Development Impede Muscle Protein Synthesis in Neonatal Pigs by Different Ribosomal Mechanisms. Pediatr Res 2011;69:473478.

31 Bruins MJ, Deutz NE, Soeters PB: Aspects of organ protein, amino acid and glucose metabolism in a porcine model of hypermetabolic sepsis. Clin Sci (Lond) 2003;104:127-141.

32 Kimball SR, Orellana RA, O'Connor PM, Suryawan A, Bush JA, Nguyen HV, Thivierge MC, Jefferson LS, Davis TA: Endotoxin induces differential regulation of mTOR-dependent signaling in skeletal muscle and liver of neonatal pigs. Am J Physiol Endocrinol Metab 2003;285:E637-644.

33 Wendorff TJ, Schmidt BH, Heslop P, Austin CA, Berger JM: The structure of DNA-bound human topoisomerase II alpha: conformational mechanisms for coordinating inter-subunit interactions with DNA cleavage. J Mol Biol 2012;424:109-124.

34 Deweese JE, Osheroff N: The DNA cleavage reaction of topoisomerase II: wolf in sheep's clothing. Nucleic Acids Res 2009;37:738-748.

35 Roca J, Wang JC: The capture of a DNA double helix by an ATP-dependent protein clamp: A key step in DNA transport by type II DNA topoisomerases. Cell 1992;71:833-840.

36 Shao Y, He J, Chen F, Cai Y, Zhao J, Lin Y, Yin Z, Tao H, Shao X, Huang P, Yin M, Zhang W, Liu Z, Cui L: Association study between promoter polymorphisms of ADAM17 and progression of sepsis. Cell Physiol Biochem 2016;39:1247-1261.

37 Silva JD, de Oliveira GP, Samary Cdos S, Araujo CC, Padilha Gde A, Costa e Silva Filho F, da Silva RT, EinickerLamas M, Morales MM, Capelozzi VL, da Silva VM, Lima LM, Barreiro EJ, Diaz BL, Pelosi P, Silva PL, Garcia CS, Rocco PR: Respiratory and Systemic effects of LASSBio596 plus surfactant in experimental acute respiratory distress syndrome. Cell Physiol Biochem 2016;38:821-835.

38 Zhang J, Zhu D, Wang Y, Ju Y: Andrographolide attenuates LPS-induced cardiac malfunctions through inhibition of IкB phosphorylation and apoptosis in mice. Cell Physiol Biochem 2015;37:1619-1628.

39 Cheng Y, Wang H, Mao M, Liang C, Zhang Y, Yang D, Wei Z, Gao S, Hu B, Wang L, Cai Q: Escin Increases the survival rate of LPS-induced septic mice through inhibition of HMGB1 release from macrophages. Cell Physiol Biochem 2015;36:1577-1586.

40 Zhu J, Duan G, Lang L, Liu Y, Zhu J, Wang H, Liu Y: The bacterial component flagellin induces anti-sepsis protection through TLR-5, IL-1RN and VCAN during polymicrobial sepsis in mice. Cell Physiol Biochem 2015;36:446-456.

41 Landgraf MA, Silva RC, Corrêa-Costa M, Hiyane MI, Carvalho MH, Landgraf RG, Câmara NO: Leptin downregulates LPS-induced lung injury: role of corticosterone and insulin. Cell Physiol Biochem 2014;33:835-846.

42 Wang M, Yan J, He X, Zhong Q, Zhan C, Li S: Candidate genes and pathogenesis investigation for sepsisrelated acute respiratory distress syndrome based on gene expression profile. Biol Res 2016;49:25. 